\title{
An outbreak of bovine trypanosomiasis in the Blue Nile State, Sudan
}

\author{
Bashir Salim ${ }^{1,2^{*}}$, Mohammed A Bakheit ${ }^{2,3}$, Sir Elkhatim Salih ${ }^{4}$, Joseph Kamau', Ichiro Nakamura ${ }^{1}$, Ryo Nakao ${ }^{1}$ and \\ Chihiro Sugimoto ${ }^{1}$
}

\begin{abstract}
Background: In this paper, we report an outbreak of bovine trypanosomiasis in Kurmuk District, Blue Nile State, Sudan that involved an infection with four Trypanosoma species in cattle. The outbreak occurred in June 2010 when indigenous cattle, mainly Kenana and Fulani breed types, crossed the national Sudanese border to Ethiopia and returned. A veterinarian was notified of massive deaths in the cattle populations that recently came from Ethiopia. All animals involved in the outbreak were from the nomadic Fulani group and resident local cattle were not infected and no death has been reported among them. A total of 210 blood samples were collected from the ear vein of cattle. A few samples were also collected from other domestic animals species. Parasitological examinations including hematocrit centrifugation techniques $(H C T)$ and Giemsa-stained thin blood films were carried out. ITS1-PCR, which provides a multi-species-specific diagnosis in a single PCR, was performed.

Findings: Parasitological examinations revealed that 43\% (91/210) of the affected cattle population was infected with two morphologically distinct trypanosomes. Seventy animals (33.3\%) were infected with T. vivax and twenty one (10\%) with T. congolense. In contrast, ITS1-PCR was able to identify four Trypanosoma species namely T. vivax, T. congolense, T. simiae and T. brucei in 56.7\% (80/141). T. brucei showed the highest prevalence of 36.9\% (52/141) and the lowest 19\% (27/141) was displayed by T. congolense. Furthermore, and because ITS1-PCR could not differentiate between T. brucei subspecies, serum resistance-associated (SRA) gene based PCR was used to detect the human T. brucei rhodesiense in T. brucei positive samples. None of the samples was shown positive for T. $b$. rhodesiense. The identity of the $400 \mathrm{bp} \mathrm{PCR} \mathrm{product} \mathrm{originating} \mathrm{from} \mathrm{T.} \mathrm{simiae,} \mathrm{was} \mathrm{further} \mathrm{confirmed} \mathrm{by}$ sequencing and subsequent phylogenetic analysis.
\end{abstract}

Conclusions: The outbreak of bovine trypanosomiasis occurred in the Blue Nile State was caused by mixed infection of two or more Trypanosoma species and the conventional parasitological examinations were not reliable in identifying all the species of Trypanosoma involved in the outbreak. It is difficult to determine the cause of the disease for the reason that the current enzootic situation in the resident cattle in the region is poorly understood. The study concluded that there are at least four species of trypanosomes that caused this outbreak in the Blue Nile State. The presence of mixed infections might have exacerbated the severity of the disease. It is hypothesized that variant parasite type(s) might have been introduced to Sudanese cattle when they crossed to Ethiopia, a tsetse belt region.

\section{Background}

Cattle population in the Blue Nile State, Sudan, is estimated to be 1,995,024 heads (Annual Report of Ministry of Animal Resources and Fisheries, Sudan, 2009). Generally, data on bovine trypanosomiasis and the vector Glossina spp. (tsetse fly) present in the Blue Nile State

\footnotetext{
* Correspondence: bashirsalim@gmail.com

'Department of Collaboration and Education, Research Center for Zoonosis

Control, Hokkaido University, Sapporo 001-0020, Japan

Full list of author information is available at the end of the article
}

and neighboring states is scarce and only one report showed the existence of Glossina fuscipes fuscipes and G. morsitans submorsitans in Kurmuk District [1].

This study has been carried out to determine the Trypanosoma species which were incriminated in an outbreak of bovine trypanosomiasis in Kurmuk District, the Blue Nile State. It is evident that disease risk depends primarily on the density of the vector and trypanosome infection rate. The affected animals were closer to Ethiopian tsetse-belt where nomads cross in with their

\section{Biomed Central}


animals/cattle in search of pasture and water. The tsetse flies in Ethiopia are confined to the southern and western regions between longitude $33^{\circ}$ and $38^{\circ} \mathrm{E}$ and latitude $5^{\circ}$ and $12^{\circ} \mathrm{N}$ [2]. This region overlaps with the area where two Glossina species were reported in the Blue Nile State, Sudan [1], though there was no report showing the tsetse belt is reaching the Blue Nile State in the Sudan.

\section{Sample collection and parasitological examination}

To preserve owners' confidentiality and to adhere to the International Ethical Guidelines for Biomedical Research involving animal subjects, no owner names were recorded within any database or as part of the data collection process. The owners of the sampled cattle provided consent to have their animals included in the study. Research on samples was conducted adhering to guidelines of the Institutional Animal Care and Use Committee of the Graduate School of Veterinary Medicine, Hokkaido University. The study protocol also followed the general guidelines for sampling domestic animals provided by the Faculty of Veterinary Medicine, University of Khartoum, Sudan.

The outbreak occurred in June 2010 (Rainy season). On the $13^{\text {th }}$ of June 2010, a total number of 210 blood samples was collected from affected cattle in Sali village at Kurmuk District $\left(10^{\circ} 78^{\prime} \mathrm{N} 34^{\circ} 18^{\prime} \mathrm{E}\right)$ in the Blue Nile State, Sudan (Figure 1). Three millilitres of blood were

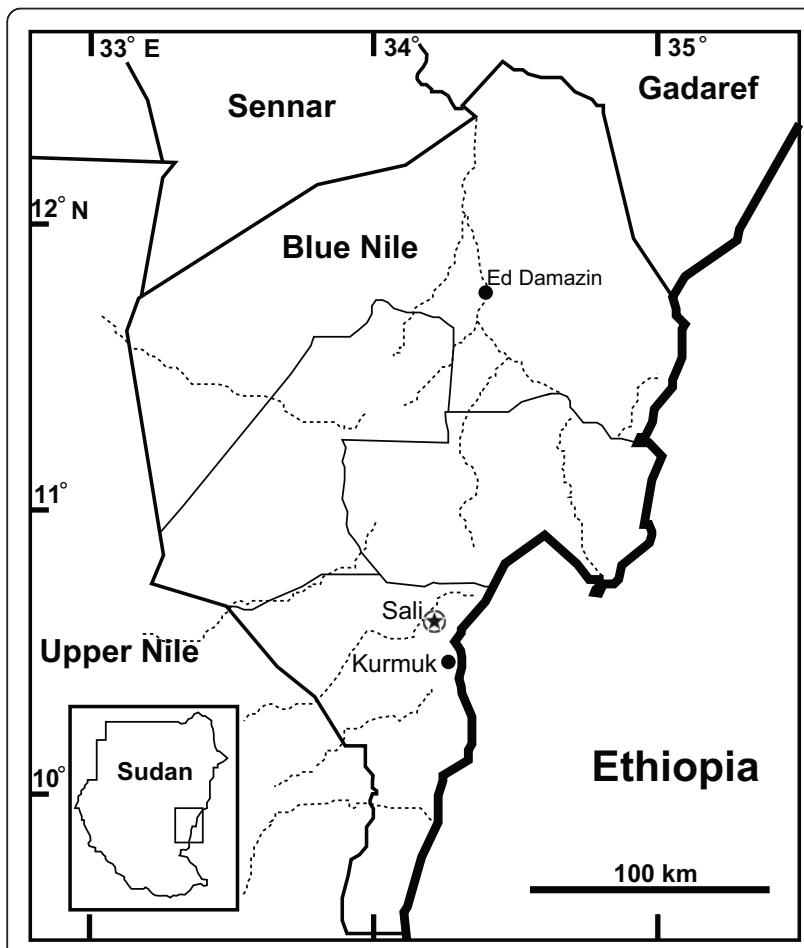

Figure 1 Map of the Blue Nile State. The outbreak location is shown in black star. collected from the ear vein in heparinized vacutainer tubes (Becton Dickinson, France). A few more samples were similarly collected from donkeys, camels and sheep in the village (Table 1). Parasitological examinations were carried out in the regional laboratory using HCT according to [3]. Subsequently, 10\% Giemsa-stained thin blood smears were prepared and examined under oil immersion field to identify trypanosomes species based on their morphological structures. For PCR, 141 samples were spotted onto FTA ${ }^{\circledR}$ elute cards (Whatman, Inc, UK), which included the 91 parasitologically positive samples and additional 50 randomly selected negative samples.

\section{DNA extraction}

Genomic DNA was extracted from six punches in each FTA card to ensure correct estimation of prevalence as described elsewhere [4-6]. Briefly, blood samples collected on FTA cards were dried thoroughly at room temperature. Using a sterile punch, each FTA card was punched out at 6 different positions each was $3 \mathrm{~mm}$ size. These were placed into a sterile microcentrifuge tube and rinsed 3 times each in $750 \mu \mathrm{L}$ of deionized water by vortexing for 5 seconds and discarding of water. DNA was eluted using a buffer that contained 90 $\mu \mathrm{L}$ deionized water plus $10 \mu \mathrm{L} 10 \mathrm{X}$ ThermoPol Reaction Buffer (Biolabs, Inc, England). Elution was performed by heating the sample at $95^{\circ} \mathrm{C}$ for 30 minutes using a heat block. Eluted DNA concentration ranged between 100 $250 \mathrm{ng} / \mu \mathrm{L}$. DNA was stored at $-20^{\circ} \mathrm{C}$ until used.

\section{PCR}

Extracted DNA was subjected to a PCR test, which amplifies the ITS1 region of the rDNA gene of all African trypanosomes by using ITS1 CF/BR CF: 5'-CCG GAAGTTCACCGATATTG-3', BR: 5'-TGCTGCG TTCTTCAACGAA-3' [7]. The 700, 480, 400, and 250 bp of PCR products corresponding to $T$. congolense (savannah), T. brucei subspecies and T. evansi, T. simiae and $T$. vivax respectively, were amplified using GoTaq ${ }^{\circledR}$ Colorless Master Mix, 2X (Promega Co., USA) in a 10 $\mu \mathrm{l}$ total volume. Each reaction included $5 \mu \mathrm{l} \mathrm{GoTaq}{ }^{\circledR}$ Colorless Master Mix, $10 \mathrm{mM}$ of each primer, $1 \mu \mathrm{l}$ RNase-free water and $2 \mu \mathrm{l}(\sim 100 \mathrm{ng} / \mu \mathrm{l})$ extracted DNA. To detect T. brucei rhodesiense, the following primer set was used FP: 5'-ATA GTG ACA AGA TGC GTA CTC AAC GC-3' and RP: 5'-AAT GTG TTC GAG TAC TTC GGT CAC GCT-3'. This primer set targets the serum resistance-associated gene (SRA) and amplifies a 284-bp fragment [8]. Thermocycling profile started with an initial hold for $2 \mathrm{~min}$ at $95^{\circ} \mathrm{C}$, followed by 35 cycles of $95^{\circ} \mathrm{C}$ for $30 \mathrm{sec}, 58^{\circ} \mathrm{C}$ for $30 \mathrm{sec}$. and $72^{\circ} \mathrm{C}$ for $1 \mathrm{~min}$. A final extension step of $5 \mathrm{~min}$ at $72^{\circ} \mathrm{C}$ ended the program. PCR products were electrophoresed in $2 \%$ agarose 
Table 1 Parasitological examinations of cattle and other domestic animals samples during an outbreak of cattle trypanosomiasis in the Blue Nile State, Sudan

\begin{tabular}{lcccl}
\hline Animal & No. samples & HCT (\%) & No. positive samples by thin film & Trypanosoma spp. identified (number, per cent) \\
\hline Cattle & 210 & 43 & 91 & T. vivax $(70,33.3 \%)$ \\
& & & & T. congolense $(21,10 \%)$ \\
Donkeys & 8 & 37.5 & 3 & T. vivax $(3,37.5 \%)$ \\
Camels & 2 & 100 & 2 & T. evansi $(2,100 \%)$ \\
Sheep & 3 & 66.7 & 2 & T. vivax $(2,66.7 \%)$ \\
\hline
\end{tabular}

Zebra (BioTools, Inc, Japan) in TAE buffer and stained using GelRed dye (Biotium, Inc., USA) before being visualized under UV light.

As T. simiae is generally assumed to be a pig pathogen, and in order to confirm the identity of the $400 \mathrm{bp}$ PCR product as originating from $T$. simiae, ITS1-PCR products obtained for three randomly selected $T$. simiae-positive samples were cleaned using Wizard ${ }^{\circledR}$ SV Gel and PCR Clean-Up System (Promega, WI, USA) and sequenced using forward and reverse primers according to the protocol of the Dye Terminator V.3.1 cycle sequencing kit (Applied Biosystem, Japan). The products were subsequently ethanol/EDTA/sodium acetate precipitated prior to loading to ABI Prism 3730 Genetic analyzer. Sequence chromatograms were edited and analyzed using the software Finch TV Version 1.4.0 (Geospiza, Inc., USA) and ApE http://www.biology.utah. edu/jorgensen/wayned/ape/. Sequences of ( 340 bp) were blasted in NCBI for sequence similarity and phylogenetic analysis to depict the relationship with other Trypanosoma spp. Sequences were deposited in the Genbank under the accession numbers [Genbank: $\mathrm{AB} 625444, \mathrm{AB} 625445$ and AB625444].

\section{Results and discussion}

The results of HCT and Giemsa-stained blood films revealed that $43 \%(91 / 210)$ of the cattle were infected with two morphologically distinct trypanosomes. Seventy (33.3\%) were infected with $T$. vivax and twenty one (10\%) with T. congolense (Table 1). The ITS1 PCR identified four Trypanosoma spp (Table 2). The mortality estimated for 4 herds (150 - 300 heads/herd) visited ranged between $20-90 \%$.

The results obtained by ITS1-PCR indicated that cattle were infected with four Trypanosoma spp. and most of the infection patterns were of mixed nature. We could detect simultaneous infection with 3 and even 4 Trypanosoma spp in a single animal (Figure 2). A total number of 80 cattle were infected, giving an overall prevalence of $56.7 \%(80 / 141)$ in these samples. $T$. b. brucei showed the highest prevalence of $36.9 \%(52 / 141)$, followed by $T$. simiae, 22\% (31/141), T. vivax, 20\% (28/141) and T. congolense, 19\% (27/141) (Table 2). It is worth noting that the PCR overall prevalence shown in this study does not constitute an accurate prevalence of trypanosomiasis in the affected population, as the majority of samples selected for PCR were known parasitologically positive. Nonetheless it is clearly reflected here that ITS1-PCR has better specificity for detection of trypanosomes. The 400 bp fragments obtained from three randomly selected T. simiae-positive samples were confirmed to be $T$. simiae by direct sequencing of the ITS1 region. The $T$. simiae detected in the in this study showed similarity to T. simiae from Kenya (accession no. TSU22320). Phylogenetic relationship of the $T$. simiae identified in this study to reference sequences of $T$. simiae, $T$ congolense and T. brucei is given in (Figure 3).

There are three known genetically distinct types of T. congolense (savannah, forest and kilifi). Only the

Table 2 Prevalence of tsetse transmitted trypanosomes as revealed by ITS1 PCR detection method in cattle samples during an outbreak of cattle trypanosomiasis in the Blue Nile State, Sudan

\begin{tabular}{lc}
\hline Trypanosoma spp. infection & Prevalence (\%) \\
\hline$T C+T b b+T S+T V$ & $1(0.7)$ \\
$T C+T b b+T s$ & $5(3.5)$ \\
$T C+T b b+T V$ & $1(0.7)$ \\
$T b b+T s+T V$ & $7(5.0)$ \\
$T S+T V+T C$ & $1(0.7)$ \\
$T C+T b b$ & $6(4.3)$ \\
$T C+T S$ & $6(4.3)$ \\
$T C+T V$ & $5(3.5)$ \\
$T b+T s$ & $5(3.5)$ \\
$T b+T V$ & $5(3.5)$ \\
$T s+T V$ & 0 \\
$T C$ & $2(1.4)$ \\
$T b$ & $22(15.6)$ \\
$T s$ & $6(4.3)$ \\
$T V$ & $8(5.7)$
\end{tabular}

Overall prevalence of each species

Tbb 36.9\% (52/141)

Ts $\quad 22 \%(31 / 141)$

TV 20\% (28/141)

Tc $\quad 19 \%(27 / 141)$

Overall prevalence of all species

$56.7 \%(80 / 141)$

Tc, Tbb, Ts and Tv represent T. congolense, T. b. brucei, T. simiae and T. vivax respectively. 


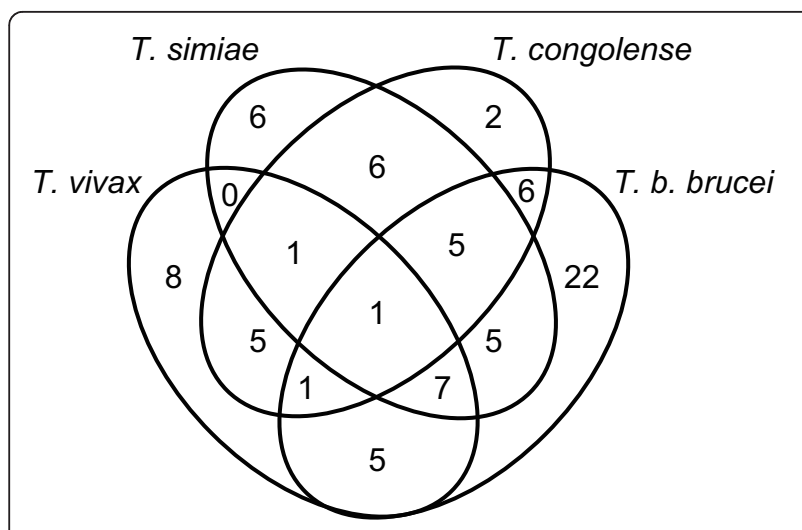

Figure 2 Single and mixed infections of four Trypanosoma spp. detected in cattle by ITS1-PCR in the Blue Nile State Sudan.

savannah type of $T$. congolense, (PCR fragment size of $700 \mathrm{bp}$ ) was detected, while the other two, $T$. congolense kilifi (620 bp) and T. congolense forest (710 bp), were not. $T$. congolense savannah is reported to be of extreme virulence [9].

The conventional parasitological methods were not able to detect and/or discriminate between $T$. simiae and T. b. brucei though the latter had the highest prevalence when we used ITS1 PCR detection methods. The low microscopic detection rate might be due to chronic enzootic nature of T. b. brucei, in which the level of parasitaemia is usually below the level of detection offered by the conventional methods. On the other hand, $T$. simiae might be misdiagnosed as $T$. congolense as the two parasites are known to be morphologically similar [10].
This study represents a first report on the presence of T. simiae in Sudan. It is worth mentioning that large numbers of wild boars (Sus scrofa) do exist in the Blue Nile State. The presence of wild boars, which graze in vicinity of domestic cattle in the region, could be linked to the presence of $T$. simiae detected in cattle during this study. It remains to be confirmed whether this animal could indeed act as a natural reservoir to this species of Trypanosoma.

Generic ITS1-PCR was developed by [11] for trypanosome detection. This method was modified to increase the sensitivity of the primers to T. vivax, an important cattle trypanosome $[7,12]$. In addition, $[13,14]$ have further refined the test to increase the sensitivity and specificity in detecting trypanosomes. The method was shown to be a powerful diagnostic technique for detecting and identifying trypanosomes quickly, accurately and cheaply. In our study, ITS1-PCR detected lesser number of positives (80) compared to the parasitological methods (91) in the same samples. Collected blood was kept in the fridge before it was spotted in the FTA cards, thus might become subjected to DNA degradation and subsequently underestimated the accurate sensitivity of the ITS1-PCR.

An additional species-specific PCR was sometimes needed when the ITS1-PCR products of some species were too similar or same in size to distinguish, for example T. brucei subspecies and T. evansi or T. simiae and T. simiae Tsavo [14]. Because ITS1-PCR could not differentiate between $T$. brucei subspecies, we used the serum resistance-associated (SRA) gene based PCR to detect the human T. brucei rhodesiense in T. brucei

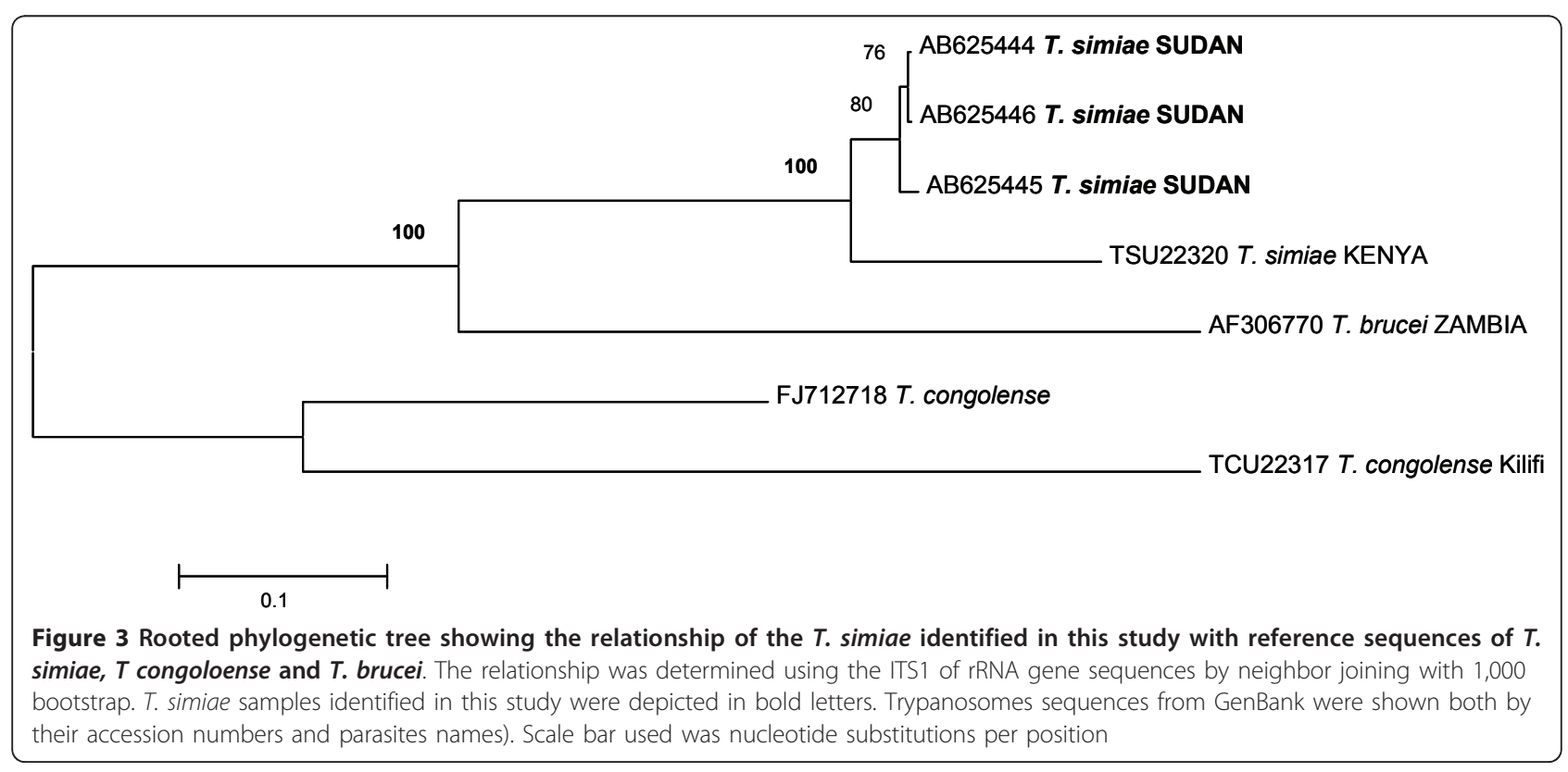


positive samples. However, none of the samples was shown positive for this species.

From the result showed in Table 1, other domestic livestock (donkeys, camels and sheep) were infected by trypanosomes based on microscopical examination. Although local resident cattle were not sampled during this study, the presence of infection in other resident animal species should imply that some if not all trypanosome species involved in this outbreak are also present in resident cattle in the region. Urgent screening of possible trypanosome species in resident cattle in the region using ITS1-PCR is required for understanding of the enzootic nature of the disease.

The study concluded that there are at least four species of trypanosomes that caused an outbreak of trypanosomiasis in cattle in the Blue Nile State. The presence of mixed infection might have exacerbated the severity of the disease. Though we hypothesized that variant parasite type(s) might have been introduced to Sudanese cattle when they cross into Ethiopia, a tsetse belt region. We conclude that future research should focus towards a better understanding of the picture of parasite endemicity for better control measures. This plan should also include surveillance for the possible Glossina and other biting fly species existing in the region.

\section{Acknowledgements \\ This work was supported by Grant-in-Aid for JSPS fellows and for Scientific Research from Ministry of Education, Culture, Sports, Science and Technology of Japan (MEXT), the program of Funding Research Center for Emerging and Re-emerging Infectious Disease, MEXT, and Asia-Africa S \& T Strategic Cooperation Promotion Program by the Special Coordination Funds for Promoting Science \& Technology, MEXT.}

\section{Author details}

'Department of Collaboration and Education, Research Center for Zoonosis Control, Hokkaido University, Sapporo 001-0020, Japan. ${ }^{2}$ Department of Parasitology, Faculty of Veterinary Medicine, University of Khartoum, 13314 Khartoum-North, Sudan. ${ }^{3}$ Veterinary Infection Biology and Immunology, Research Center Borstel, D-23845 Borstel, Germany. ${ }^{4}$ Damazin Veterinary Laboratory, Blue Nile State, Federal Ministry of Animal Resource and Fisheries, Sudan.

\section{Authors' contributions}

BS carried out the molecular genetic analyses, participated in the data analysis, did the field collection, participated in the statistical analysis and drafted the manuscript. MAB was involved in field collection and helped to draft the manuscript. SS performed parasitological examination and involved in field collection. JK, IN and NR participated to draft the manuscript. CS helped to conceive the study, participated in its design, assisted in obtaining funding and helped to draft the manuscript. All authors read and approved the final manuscript.

\section{Competing interests}

The authors declare that they have no competing interests.

Received: 18 March 2011 Accepted: 13 May 2011

Published: 13 May 2011

\section{References}

Mahamed-Ahmed MMahamed (1989) Distribution of Tsetse in Kurmuk District Blue Nile Province, Sudan. Sud J Vet Sci Anim Husb 28:1
Abebe G (2006) Trypanosomiasis. The Epidemiology and Ecology of Health and Disease in Ethiopia. Shama Books. Addis Ababa, Ethiopia

Woo PTK (1971) Evaluation of heamatocrit certifuge and other techniques for field diagnosis of trypanosomiasis and filariasis. Act Trop 28:298-303

Cox AP, Tosas O, Tilley A, Picozzi K, Coleman P, Hide G, Welburn SC (2010) Constraints to estimating the prevalence of trypanosome infections in East African zebu cattle. Parasit Vectors 3:82. doi:10.1186/1756-3305-3-82.

Salim B, Bakheit MA, Kamau J, Nakamura I, Sugimoto C (2011) Molecular epidemiology of camel trypanosomiasis based on ITS1 rDNA and RoTat 1.2 VSG gene in the Sudan. Parasit Vectors 4:31. doi:10.1186/1756-3305-4-31.

Ahmed AH, MacLeod TE, Hide G, Welburn CS, Picozzi K (2011) The best practice for preparation of samples from FTA $\mathrm{F}^{\oplus}$ cards for diagnosis of blood borne infections using African trypanosomes as a model system. Parasit Vectors 4:68. doi:10.1186/1756-3305-4-68.

Njiru ZK, Constantine CC, Guya S, Crowther J, Kiragu JM, Thompson RC, Dávila AM (2005) The use of ITS1 rDNA PCR in detecting pathogenic African trypanosomes. Parasitol Res 95:186-192. doi:10.1007/s00436-004-1267-5.

Radwanska M, Chamekh M, Vanhamme L, Claes F, Magez S, Magnus E, de Baetselier P, Büscher P, Pays E (2002) The serum resistance-associated gene as a diagnostic tool for the detection of Trypanosoma brucei rhodesiense. Am J Trop Med Hyg 67:684-690

Bengaly Z, Sidibe I, Boly H, Sawadogo L, Desquesnes M (2002) Comparative pathogenicity of three genetically distinct Trypanosoma congolense-types in inbred Balb/c mice. Vet Parasitol 105:111-8. doi:10.1016/50304-4017(01) 00609-4.

Majiwa PA, Webster P (1987) A repetitive deoxyribonucleic acid sequence distinguishes Trypanosoma simiae from T. congolense. Parasitol 3:543-58

Desquesnes M, McLaughlin G, Zoungrana A, Davila AMR (2001) : Detection and identification of Trypanosoma of African livestock through a single PCR based on internal transcribed spacer 1 of rDNA. Int J Parasitol 31:610-614. doi:10.1016/50020-7519(01)00161-8.

Njiru ZK, Makumi JN, Okoth S, Ndungu JM, Gibson WC (2004) Identification of trypanosomes in Glossina pallidipes and G. Iongipennis in Kenya. Infect Genet Evol 1:29-35

Cox A, Tilley A, McOdimba F, Fyfe J, Eisler M, Hide G, Welburn S (2005) A PCR based assay for detection and differentiation of African trypanosome species in blood. Exp Parasitol 1:24-9

Adams ER, Malele II, Msangi AR, Gibson WC (2006) Trypanosome identification in wild tsetse populations in Tanzania using generic primers to amplify the ribosomal RNA ITS-1 region. Acta Trop 1-2:103-9

doi:10.1186/1756-3305-4-74

Cite this article as: Salim et al:: An outbreak of bovine trypanosomiasis in the Blue Nile State, Sudan. Parasites \& Vectors 2011 4:74.

\section{Submit your next manuscript to BioMed Central and take full advantage of:}

- Convenient online submission

- Thorough peer review

- No space constraints or color figure charges

- Immediate publication on acceptance

- Inclusion in PubMed, CAS, Scopus and Google Scholar

- Research which is freely available for redistribution

Submit your manuscript at www biomedcentral com/submit
Ciomed Central 